\title{
Les enjeux psychologiques de la mise à distance en formation
}

\author{
Jean-Claude Maurin \\ Psychosociologue, \\ Promoteur et animateur du dispositif européen de formation de Formateurs en \\ réseau Net-Trainers \\ jeanclaude.maurin@free.fr
}

RÉSUMÉ. La présentation d'une expérience de formation de formateurs à distance permet de montrer la possibilité de préserver une certaine qualité de relation humaine, basée sur l'approche rogérienne, dans ce contexte d'éloignement physique. Pour prolonger ce témoignage, l'auteur tente d'identifier les difficultés psychologiques qui s'opposent à une mise à distance de la relation pédagogique. Cette mise à distance amène en effet des espérances chez certains professionnels de la formation et des résistances chez d'autres. Certains apports de la théorie psychanalytique, de même qu'ils éclairent les enjeux de la relation pédagogique, peuvent permettre de mieux comprendre ces résistances.

ABSTRACT. This article is based on a specific experience with an online training course for trainers. It illustrates the possibility to retain, in the context of distance learning, quality interpersonal relationships using the approach developed by Rogers. The author identifies the psychological factors which account for the difficulties in dealing with and accepting a distant pedagogical relationship. For training or teaching professionals, the development of distance learning has indeed led to rising hopes but also to growing resistance. Some elements of the theories of psychoanalysis can provide an interesting outlook on what is at stake in a pedagogical relationship and contribute to our understanding of this resistance.

MOTS-CLÉS : relation pédagogique, formation ouverte et à distance, formation de formateurs, écoute, congruence, transfert, dynamique de groupes, fantasme, résistance.

KEYWORDS: pedagogical relationship, open and distance learning, training for trainers, listening, congruence, transference, group dynamics, fantasy, resistance.

D\&S - 2-3/2004. Enigmes de la relation pédagogique, pages 183 à 204 


\section{Introduction : la mise à distance interroge la relation}

Dans ma pratique de formateur et d'intervenant dans le domaine de la formation ouverte et à distance (FOAD), j'ai l'occasion de rencontrer de nombreux professionnels de la formation et de l'éducation. Beaucoup parmi eux s'interrogent sur la possibilité d'établir une relation pédagogique, ou de préserver celle-ci, dans le cadre de la formation à distance. J'essaie alors d'expliquer avec pragmatisme que la mise à distance ne constitue pas véritablement une difficulté. Dans ma propre expérience en effet, il est devenu « évident» que des relations humaines de qualité peuvent s'établir avec des apprenants distants. Lorsqu'ils participent aux formations de formateurs que je propose, ces professionnels peuvent eux-mêmes découvrir et expérimenter la possibilité d'établir un climat relationnel riche et productif malgré l'éloignement physique. Mais il ne s'agit pas là d'une vérité universelle et cet article permettra de questionner et de relativiser cette affirmation. De plus, ce témoignage ne suffit pas, loin s'en faut, à surmonter les hésitations ou les oppositions que les professionnels manifestent pour s'engager eux-mêmes dans la mise à distance de leur formation. Lors des conférences, des réunions ou des sessions de formation sur la FOAD dans lesquelles j'interviens, je rencontre aussi du scepticisme; je peux entendre des objections sur la pertinence, par exemple, à développer la FOAD avec tel ou tel public qui aurait des besoins relationnels particuliers et ne serait pas, $a$ priori, autonome. Les professionnels expriment des craintes, ainsi que le besoin d'être rassurés avant de faire «le grand saut» de la mise à distance. Ces réactions m'interrogent à double titre. J'ai le plaisir de partager mon enthousiasme pour l'ouverture des systèmes de formation avec d'autres formateurs et promoteurs de projets, mais j'ai aussi l'impression que nous appartenons à un cercle restreint. J'en viens alors à douter de mes capacités à partager mes convictions, mais aussi à soupçonner chez d'autres des signes de résistance ${ }^{1}$. Je dois cependant reconnaître, avec Mireille Cifali (1994) que « la résistance que l'on pointe chez l'autre s'assortit d'un rejet dès lors qu'on ne souscrit pas à ce qui est proposé. C'est lui qui a tort, qui ne comprend pas ; il est particulièrement stupide, rétif ou conservateur. La résistance de l'un met l'autre en situation d'impuissance». Cette résistance s'explique pourtant : c'est du moins ce que je voudrais tenter de faire ici. Elle «n'est nullement une réalité négative, elle a son intelligence, elle représente un défi pour la pensée ».

Derrière les débats que suscite la mise à distance et les problèmes qu'elle pose, ne voit-on pas se réactiver des alternatives qui agitent le monde de l'éducation et de la formation depuis de nombreuses années? Je suis pour ma part engagé dans des projets et des activités de FOAD depuis une décennie avec la forte intuition que les nouvelles technologies numériques ne constituent pas, en tant que telles, l'élément déterminant d'une transformation de la relation pédagogique. Comme le précise en effet Michel Bernard (1999), «La question de la distance traverse la formation. Elle

1. Le terme de résistance est utilisé ici dans son sens psychologique et il sera défini dans le chapitre «L'importance du tiers dans la mise à distance » ci-après. 
est bien antérieure aux apports et usages [de ces technologies]. L'oublier, c'est conférer à certains aspects de la distance un poids exagéré et par ailleurs, minimiser d'autres aspects de la pluralité de la distance. » Nous sommes donc face à un enjeu qui dépasse largement les considérations géographiques et techniques. La relation pédagogique concentre les passions et les tensions ; il n'est donc pas surprenant que la perturbation apportée dans ses modalités vienne susciter certaines réactions de défenses. Comme le précise encore Mireille Cifali (1994), les résistances indiquent à chaque fois « qu'on touche à quelque chose de sensible, d'intolérable, que le moi est mis en danger ». De plus, ces enjeux psychologiques font écho à des considérations d'ordre politique. Pour de nombreux auteurs en effet (Collectif de Chasseneuil, 2001), il s'agit bien avec la FOAD de donner une place plus centrale à l'apprenant et de valoriser son autonomie. L'accent mis sur l'apprentissage et l'autodirection de celui-ci ne vient-il pas déplacer les lignes du pouvoir et menacer des modèles profondément ancrés, psychologiquement structurants, qui privilégient l'enseignement magistral ainsi que l'autorité dans la relation pédagogique ?

Nous tenterons d'abord de répondre à la question : est-il possible d'instaurer une relation pédagogique à distance ? Nous proposerons pour cela un témoignage basé sur notre pratique. Dans le contexte décrit, celui de la formation de formateurs que nous conduisons, nous présenterons des exemples de processus relationnels observés. Nous interrogeant sur les possibilités de généraliser une telle expérience, nous nous demanderons ensuite si les conditions d'une bonne relation interpersonnelle peuvent être réunies dans la formation à distance. Si les difficultés identifiées dans la mise à distance ne suffisent pas à expliquer les résistances que nous soupçonnons, il conviendra de situer plus précisément le problème sur un terrain psychologique. Cette réflexion sur ce qui constitue la relation nous amènera ainsi à adopter un point de vue paradoxal : d'une certaine manière, la distance est inhérente à la relation, car il n'y a pas de relation sans séparation. Dans une telle perspective, les résistances opposées à la mise à distance prennent du sens. L'éloignement physique n'entraine pas systématiquement un éloignement psychologique entre apprenants et formateurs. Il peut quelquefois empêcher, nous le verrons, que la relation entre eux ne soit exclusive ou fusionnelle.

\section{Le dispositif de formation de formateurs à distance}

Nous présentons ici une expérience et une pratique sur laquelle s'appuient nos observations, pour illustrer les possibilités et les conditions de mise en place d'une relation pédagogique à distance.

Les participants à cette formation de formateurs en ligne ${ }^{2}$ ont des profils divers : formateurs en centres de formation intervenant dans différents domaines (langues, informatique, secteur industriel, services, management, insertion professionnelle,

2. La présentation de cette formation est disponible à l'adresse : <www.nettrainers.org/fr> 
etc.) ; enseignants du public et du privé ; formateurs en entreprise (dans l'industrie, la banque, les assurances, etc.) ; responsables de formation ou consultants. Ils sont salariés à temps plein, à temps partiel, indépendants ou demandeurs d'emploi.

Cette formation vise le développement de compétences transversales permettant aux participants de conduire et de développer des formations en réseau dans leur domaine de spécialité. Pour accéder à cette formation, les participants doivent maîtriser l'usage simple du traitement de texte, l'usage de la messagerie électronique et des principes de la navigation internet, disposer d'un accès à un ordinateur équipé d'une connexion internet. Ils doivent avoir une expérience minimale de un an dans la formation ou l'enseignement, et disposer d'une disponibilité moyenne de 7 à 10 heures par semaine, pour une durée totale de 160 heures.

L'approche proposée dans cette formation de formateurs affiche 7 principes :

L'ouverture : elle est réalisée entièrement à distance et par internet, selon des modalités de communication essentiellement asynchrones, afin d'autoriser une souplesse maximale. Des participants du monde entier se forment à leur rythme, à leur domicile ou sur leur lieu de travail.

L'accompagnement: le dispositif propose un tutorat personnalisé intensif. Durant toute la durée du parcours, des formateurs spécialistes et expérimentés soutiennent la progression de chaque participant. Ils fournissent une évaluation continue des travaux ainsi que du conseil et de l'assistance rapide.

La compétence : chaque participant est accompagné jusqu'à ce qu'il ait atteint les objectifs pédagogiques du programme. Les méthodes d'évaluation garantissent l'acquisition d'un niveau de compétence conforme au référentiel professionnel de « formateur en réseau ».

Le contrat: le participant élabore avec son formateur, dans un principe de réciprocité, un contrat pédagogique basé sur ses besoins, attentes, priorités, ainsi que sur sa situation professionnelle et personnelle. Le participant et son formateur définissent ensemble leurs engagements respectifs, un calendrier de travail et des procédures de communication.

L'apprentissage expérientiel: les participants se forment à la FOAD en pratiquant eux-mêmes la situation d'apprenants distants en ligne. Ils réalisent de nombreuses activités d'apprentissage qui les placent également en position de formateur en réseau ou de concepteur de cours en ligne.

Le projet: les contenus de la formation font alterner des éléments de méthodologie générale et le développement de leur propre projet de formation en réseau.

L'approche collaborative: un certain nombre d'activités d'apprentissage aboutissent à des productions collectives et/ou reposent sur la communication entre pairs, au sein de petits groupes de travail. Les participants sont ainsi amenés à partager leurs expériences et à échanger sur leurs pratiques. 
Un climat relationnel favorable est recherché, dans cette formation, par la mise à disposition de quelques outils de communication simples, mais surtout au moyen de dispositifs ou de situations pédagogiques appropriés.

\section{Le contrat pédagogique}

C'est un outil pédagogique destiné à clarifier les engagements réciproques des acteurs de la formation. Il a une valeur morale et précise ce que les participants et les formateurs peuvent attendre les uns des autres dans ce contexte de formation à distance. Ici en effet, la «présence» dans la formation se traduit par des productions et des messages, par des traces essentiellement écrites. Le contrat fournit des repères utiles dans le déroulement du parcours de formation et dans l'évaluation de ses conditions de réalisation. Il n'entraîne pas de " sanctions » autres que des échanges de clarifications. La souplesse possible dans l'aménagement du parcours de formation implique une nécessaire rigueur dans les engagements de part et d'autre.

Il est conçu dans la perspective que proposait Rogers (1999) : «Un dispositif ouvert qui donne à la fois une certaine sécurité et une réelle responsabilité dans une ambiance de liberté [...] Il n'est pas douteux, d'ailleurs, que ce système calme dans une certaine mesure les appréhensions et les incertitudes du facilitateur ${ }^{3}$. »

La réciprocité des engagements et leur personnalisation permettent d'éviter un formalisme qui viderait ce contrat de sa substance et lui assurent, au contraire, d'être utilisé tout au long de la formation.

\section{Les activités collaboratives}

Les participants sont souvent amenés, au cours de cette formation, à partager des réflexions et des informations avec d'autres participants de leur sous-groupe, mais aussi à coopérer pour la réalisation de certaines activités d'apprentissage.

Des outils de communication (forum, chat) permettent les échanges au sein du grand groupe ou des petits groupes. Chacun peut aussi utiliser ces outils pour la communication "privée » avec les autres participants. Les petits groupes sont composés de 5 à 8 participants. Les formateurs proposent une première composition de ces sous-groupes, mais les participants ont ensuite la possibilité de choisir, en tenant compte de critères tels que : les affinités personnelles, les complémentarités professionnelles ou les contraintes de gestion du temps. La constitution de ces sousgroupes est dans un premier temps liée au fait de faire connaissance par un premier échange intensif.

3. Carl Rogers substitue ici le terme de «facilitateur» à celui «d'enseignant», dans la mesure où son propos se situe dans une perspective où prédomine le processus « apprendre » et non le processus « enseigner». 


\section{L'authenticité (induite par les présentations)}

$\mathrm{Au}$ début de la formation, chacun doit envoyer par courrier électronique sept questions à l'un des autres acteurs (participant ou formateur) dans le but de le connaître mieux et de faciliter sa présentation personnelle. Chacun doit alors se présenter à lui en répondant à ses questions. Chaque acteur du dispositif doit garder à l'esprit que ses réponses seront très utiles pour les autres membres du groupe et lui permettront d'être identifié au sein du groupe. Lorsque quelqu'un a reçu les réponses de son pair, il doit les lire soigneusement et s'assurer d'avoir bien compris sa présentation. Quand tout sera clair, il introduira cette personne en envoyant sa présentation, par courrier électronique, à tous les autres membres du groupe et aux formateurs. Dans un tel protocole, le ton employé par les formateurs ou certains participants sera déclencheur d'une plus ou moins grande authenticité ; cet enjeu est généralement bien perçu et on ne cherchera pas à gêner ceux qui ont besoin de protéger leur image. Il suffit que le processus s'installe et un climat d'authenticité peut se développer progressivement au sein du groupe.

\section{Des processus relationnels significatifs}

Qu'observons-nous dans le dispositif de formation de formateurs précédemment décrit? Des processus relationnels se produisent-ils dans ce groupe «virtuel», parmi des individus éloignés dans un rayon de plusieurs centaines de kilomètres ? Nous tentons de rendre compte de leur manifestation entre participants et formateurs d'une part et, d'autre part, parmi les participants eux-mêmes.

\section{Les processus de transfert}

Lorsqu'ils s'expriment dans le contexte de la formation, les sentiments, tels que l'amour ou la haine, ne sont pas véritablement destinés aux acteurs de la formation eux-mêmes. Ils sont détournés, pourrait-on dire, de leurs objets primordiaux: généralement la mère ou le père, «bons » ou « mauvais » parents, dont le formateur prendrait la place dans l'inconscient du sujet en formation. Que pouvons-nous observer, dans notre pratique de formation à distance, en ce qui concerne ces processus de transfert entre participants et formateurs? D'abord un cas de reconnaissance enthousiaste.

Une participante écrit, à la fin d'un document de travail destiné à son formateur : " Merci à toute l'équipe à l'origine de ce projet de formation et plus particulièrement à $X$ [le formateur homme], que je n'ai guerre remercié jusqu'à ce jour tant la formation m'a envahie!!! Et oui ! Revers de la médaille, c'est tellement passionnant que l'on en oublie l'attention à porter aussi à son super formateur!!! Je comble donc mes déficiences: Merci X, Merci X, Merci X, Merci X, Merci X, Merci X.» 
Cette personne produit elle-même un considérable effort d'apprentissage, en comparaison duquel l'apport de contenu formatif peut sembler minime. Cet effort est exprimé par la métaphore guerrière de l'invasion, elle-même renforcée par une faute d'orthographe malencontreuse («je n'ai guerre remercié») et par l'évocation de cette «médaille» qu'elle a sans doute bien méritée. Mais le théâtre militaire laisse place à la bataille amoureuse puisque, grâce au «super formateur », la voici comblée et « envahie» par cette formation « tellement passionnante». Le formateur ne peut que lui témoigner des félicitations, mais quelle place occupe-t-il dans cette manifestation d'amour qu'il reçoit en retour?

Le processus de transfert se manifeste aussi par des marques de confiance et le fait de se confier. Le formateur se voit investi d'une capacité de recevoir, de contenir et de porter des révélations essentielles dans la vie d'un participant. Faisant suite à une production relative au projet professionnel, le formateur est amené à manifester son écoute au moyen d'un simple message dans lequel il écrit : "j'ai cru sentir une situation professionnelle un peu délicate. Souhaites-tu m'en dire plus, sous couvert de confidentialité, bien sûr ? » Peu de temps après, il reçoit une longue réponse dans laquelle sont détaillées les péripéties qui ont amené le participant à perdre son emploi, les divergences idéologiques profondes qui l'ont opposé à son employeur, mais aussi la longue maladie dont il est affecté et dont il est difficile de parler : " $j$ 'ai été touché par ta marque de sympathie, je t'en remercie, j'ai rarement avoué cette maladie uniquement à des personnes de confiance. » Ce que le participant exprime ici de son ressenti, ce qu'il dévoile et qu'il confie déborde en effet de la simple question posée, de sorte que celui auquel cela s'adresse représente plus et autre chose qu'un formateur.

On considère généralement que, lorsqu'une relation est engagée, les mécanismes inconscients de recherche d'appartenance, de désir de reconnaissance, de peur de l'incompréhension ou du rejet par l'autre vont se mettre en œuvre; les sentiments d'amour et de haine vont prendre place dans l'expérience subjective des sujets en interaction. Il s'agit de parvenir à une certaine proximité, à un certain degré de ressenti de l'autre qui expose inévitablement à une éclosion de sentiments. La relation pédagogique n'échappe pas à ce phénomène de transfert: même si le professionnel de la formation se défend d'éprouver des sentiments, il ne peut éviter d'en être la cible de la part des apprenants (Cifali, 1994).

Le transfert se définit, en psychanalyse, comme une expression de l'affectivité du patient à l'égard de l'analyste. «Freud l'a désigné. Il s'agirait d'un amour venant là où il n'aurait rien à y faire : dans la relation professionnelle. » (Cifali, 1994) Il s'agit en fait d'un déplacement des sentiments, des désirs inconscients qui trouvent à s'exprimer dans ce cadre-là, mais dont l'origine se trouve tout à fait ailleurs, dans l'histoire personnelle du sujet. Un aspect important du rôle du psychanalyste est justement de bien comprendre que ces manifestations amoureuses ou bien hostiles ne lui sont pas destinées, mais qu'elles représentent des moments essentiels dans le processus thérapeutique. La prise de conscience de ce qui s'actualise dans le transfert constitue même le but de l'analyse, la connaissance de soi que peut y 
rechercher le sujet. Mais le transfert comporte aussi un véritable danger, car « toute manipulation mentale passe par cet accrochage [...] Celui qui est l'objet d'un transfert d'amour a un pouvoir exorbitant. » (Cifali, 1994) Si cette notion de transfert n'est pas toujours familière aux professionnels de la formation, il est par contre clair, du point de vue des psychanalystes eux-mêmes, que ce phénomène concerne tous les métiers basés sur les relations humaines. Pour Lacan en effet, « chaque fois qu'un homme parle à un autre de façon authentique et pleine, il y a, au sens propre, transfert » (cité par Cifali, 1994).

La prise en compte du transfert éclaire mieux la menace que fait planer la relation pédagogique pour un professionnel de la formation. D'une part, elle présente un risque car elle peut amener des émotions douloureuses, de la haine, du rejet. Elle suscite donc des craintes, peut aussi être fuie ou niée dans une illusoire neutralité affective. Les manifestations du transfert font à la fois la difficulté et l'efficacité de la relation pédagogique. Elles témoignent que l'accrochage relationnel se réalise bien, mobilisant une énergie favorable à l'apprentissage, mais elles ne constituent pas une fin en soi. A l'opposé de la fuite ou de la pseudo neutralité, la quête d'amour ne saurait représenter pour le formateur un objectif professionnel. Il est donc important de ne pas focaliser les relations pédagogiques seulement sur une relation duelle, ni de réduire celles-ci au couple formateur-apprenant.

\section{La dynamique des groupes à distance}

Les relations entre pairs apprenants ouvrent en effet une autre possibilité de mise en jeu de la proximité/distance psychologique. Pour ce qui nous a été donné d'observer, tous les groupes en formation à distance ne connaissent pas d'histoires tumultueuses et passionnées; pas plus d'ailleurs que les groupes participant à des formations traditionnelles. Beaucoup d'entre eux réalisent les tâches collaboratives en limitant leur expérience à des contacts fonctionnels ; ils maintiennent une certaine retenue dans l'expression de critiques ou de réprobations même si, par exemple, l'investissement de chaque membre dans les travaux est très inégal. Dans ces groupes, le leadership est peu pris en charge en interne et, malgré la présence d'un pair coordonnateur, on attend du formateur qu'il assume les fonctions de régulation légitimées par son statut.

Voici un exemple de message, adressé à ses pairs peu actifs, par une participante chargée de la coordination d'une activité collective: "Je pense que nous devons nous entraider, néanmoins, il faut aussi que les personnes qui ont besoin d'aide face un signe: pour qu'il y ait une Bonne réponse, il faut une question! Je ne peux que vous encourager à maintenir un rythme dans votre travail et de nous faire part de vos avancements afin de vous proposer notre appui lorsque c'est possible et aussi que l'ensemble du groupe puisse s'organiser ! »

Certains participants expriment individuellement des regrets auprès du formateur, vis-à-vis de ce qu'aurait pu apporter une collaboration plus intense, mais 
personne ne prendrait le risque de menacer la cohésion du groupe par des remises en cause aux issues incertaines.

On assiste dans d'autres cas à des expériences d'une intensité affective plus forte. Les espaces de communication assignés (forum et messagerie notamment) sont abondamment investis; les discussions sur les modalités de collaboration peuvent prendre une très large place. Des leaders peuvent ainsi être investis positivement sur de longues durées. Leur groupe recherche alors des niveaux de qualité dans les productions qui dépassent souvent les critères assignés. Ce type de groupe, nous l'avons observé dans ce dispositif particulier, est très avide de gratifications : les renforcements positifs, les félicitations ou les remerciements s'échangent abondamment entre les membres.

Les extraits de messages ci-après témoignent des caractéristiques de ce groupe actif, sous l'influence d'un leader positif.

Message 1: «A mon tour de témoigner et de vous remercier. J'ai réellement beaucoup apprécié les travaux que nous avons pu faire ensemble. Certaines productions sont vraiment très intéressantes mais surtout elles ont été faites dans une ambiance très positive malgré les difficultés passagères des uns et des autres. Cela a été pour moi primordial, si l'ambiance n'avait pas été celle-la, si ça n'avait pas été vous, c'est certain j'arrêtais en cours de route. »

Message 2 : "BRAVO !! ET MERCI POUR VOTRE SOLIDARITE ET VOTRE EFFICACITE! J'ai vraiment été très heureuse de partager cette expérience avec vous; sans vous ce parcours de formation aurait été beaucoup plus laborieux. Je voudrais aussi dire merci à X [participante assumant le leadership du groupe] pour son efficacité dans la coordination du groupe et son humour. »

Message 3 : «L'équipe [nom du groupe], a été une expérience enrichissante tant dans les productions que nous avons construites que dans les communications... Merci aussi à X qui aura été tour à tour humoriste, encourageante, solidaire.... Prête, sûre à assurer une formation en réseau dès demain. Et comme l'a écrit [une autre participante], sans vous tous, le parcours aurait été plus laborieux, moins riche... »

La mobilisation dans la communication, dans ce cas, déborde largement du cadre de la formation. Des groupes de diffusion et des chats se créent à l'extérieur des espaces de communication fournis par le dispositif de formation « officiel». Les temps consacrés à ces échanges peuvent ainsi empiéter considérablement sur les temps de vie privée, le plus souvent donc, en période nocturne. Deux remarques se dégagent de l'observation d'une telle dynamique. Le degré d'appropriation et d'atteinte des objectifs d'apprentissage est ici très élevé. Les participants manifestent une forte ambition d'approfondir les thèmes de la formation. Leurs propres recherches alimentent le groupe par des apports de contenus qui dépassent souvent ce que les formateurs seraient eux-mêmes capables d'apporter. D'autre part, de telles dynamiques en viennent à exclure (courtoisement) le formateur ; ce dernier 
est utilisé pour fournir les avis ou les conseils qui lui sont demandés. Compte tenu $\mathrm{du}$ niveau d'investissement des participants, ces demandes sont d'ailleurs exigeantes, puisqu'il s'agit de donner le change. Ainsi le formateur peut apporter des gratifications et en recevoir en répondant à ces demandes, mais il serait certainement malvenu de sa part de chercher «à trop en faire », sans prendre le risque de casser une dynamique manifestement propice à l'apprentissage.

Cette dernière remarque vaut probablement aussi face à d'autres expériences de groupe, plus difficiles. Nous avons assisté dans ce contexte de mise à distance, comme cela peut se produire dans d'autres situations de formation traditionnelles, à des phénomènes de rejet ou de conflit. Ici aussi les divers moyens de communication à distance, « officiels » ou non, sont abondamment investis pas des participants qui ont pu aller jusqu'à échanger «à boulets rouges » durant tout un week-end. Dans ce cas particulier, le conflit de pouvoir au sein du groupe s'est nourri d'un désaccord sur le mode de coordination exercé par l'un des membres. Il s'est exprimé par des manifestations émotionnelles intenses : pleurs au téléphone, menaces d'abandonner la formation. Face à cela, le formateur est garant du bon fonctionnement de la communication et de la préservation des chances de chaque participant de réussir son parcours de formation. Il pourrait avoir l'ambition de réguler lui-même les échanges, de stopper les possibles débordements, sans en avoir forcément les moyens. Il est certainement possible, en formation à distance, de circonscrire moralement et techniquement la communication, en imposant aux participants un usage exclusif de certains canaux, comme par exemple un forum régulé par le formateur lui-même. Mais si tel n'est pas le cas, s'il n'y a pas de règles strictes empêchant les échanges «parallèles" (téléphones personnels, groupes de discussions privés sur internet, etc.), le formateur se voit obligé d'accepter le destin imprévisible de tout processus relationnel. Il semble alors que ces processus se développent, échappant pour une bonne part à sa maîtrise, s'il est justement prêt à accepter de ne pas les soumettre à son contrôle. Dans le cas évoqué ici, il a pu intervenir en proposant des modalités de régulation du conflit, mais le groupe a expressément choisi de le maintenir à l'écart de certaines des discussions.

Il est intéressant pour nous d'observer que de telles relations peuvent se produire dans le contexte de formation à distance et qu'elles prennent corps dans un processus de groupe. En accord avec le propos de Max Pagès (2002), on observe en effet que les membres du groupe partagent une «expérience affective de la relation» qui ne les relie pas seulement «à tel être particulier, mais à tous». La dynamique du groupe est mobilisée par une tension psychologique union/séparation parmi ses membres: "l'angoisse de la solitude, la solitude et la séparation éprouvées comme une souffrance, sont déjà le signe d'un lien avec les autres, elles portent en elles la conscience obscure d'une solidarité ». 


\section{Nouvelles interrogations sur les difficultés de la mise à distance}

Malgré le caractère particulier de la pratique dont nous venons de témoigner, il semble possible de soutenir que celle-ci est comparable à d'autres expériences de relation pédagogique, elles aussi à distance, réalisées dans différents contextes d'enseignement ou de formation professionnelle et universitaire. Nous montrons avec de tels témoignages que des processus relationnels peuvent très bien se produire dans un contexte d'éloignement géographique, entre des personnes qui ne se sont jamais rencontrées physiquement. Comment expliquer alors les difficultés auxquelles se heurte le développement de la formation à distance dans d'autres projets?

Certaines d'entre elles ont un caractère objectif et elles sont d'ailleurs souvent invoquées; nous pensons notamment au manque de moyens économiques et techniques. Selon des observations issues de notre participation active dans une dizaine de projets de développement de la FOAD, le manque de moyens matériels ne semble pas constituer une explication sérieuse. Ces projets, en effet, s'inscrivent pour la plupart dans le cadre de programmes européens et disposent donc de ressources matérielles susceptibles de satisfaire à leurs besoins économiques et techniques.

Qu'en est-il alors du facteur humain ? Les acteurs de la formation perçoivent-ils a priori la mise à distance comme une mutilation de la relation pédagogique ? Le terme de « distance physique », souvent employé pour spécifier ce contexte et cette modalité de formation, ne laisse-t-il pas entendre que la relation, en situation de face à face pédagogique, serait " réchauffée " par la proximité ou même le contact physique ? Face à l'attente ou au besoin de proximité physique dans un dispositif de formation, il est difficile de faire la part entre les nécessités objectives d'un lien favorable à l'apprentissage et les besoins subjectifs éprouvés par les acteurs.

Ce questionnement peut émerger grâce au détour de la mise à distance. Il reste enfoui et se pose rarement si l'on ne sort pas du cadre traditionnel du face à face pédagogique. Implicitement, dans ce contexte, la mobilisation globale des cinq sens dans la communication pédagogique constitue une évidence. C'est ce que suggère l'idée de proximité, de «chaleur humaine». Ne faut-il pas alors, face à une telle évidence, effectuer un deuxième détour et se demander ce qui peut se passer lorsque l'une ou l'autre des capacités sensorielles vient à manquer? Notre parcours professionnel nous a amené à vivre et à expérimenter des relations pédagogiques avec des apprenants déficients sensoriels, ou encore dans d'autres contextes culturels. Nous pouvons tenter d'en tirer ici quelques enseignements, notamment quant à ce qui est physiquement, sensoriellement nécessaire pour établir un lien pédagogique. Après ce deuxième détour, ne serons-nous pas amenés à considérer cette idée commune, celle de la nécessité du contact physique, comme une représentation fantasmée de la relation pédagogique? 


\section{Réflexions autour de la privation sensorielle}

Dans le contact physique, il s'agit en effet de voir, d'entendre, de toucher, sentir et, plus exceptionnellement dans le contexte de la formation, de goûter! Les observations suivantes tentent de faire la part entre des besoins raisonnés et des attentes plus ou moins conscientes.

\section{Voir et paraître}

Certaines de nos expériences de formation en ligne auprès d'apprenants aveugles montrent la nécessité toute relative de «voir» dans la conduite d'une formation efficace. Il s'agit là d'une situation révélatrice quant à l'intérêt réel de l'usage de la vue. Pour ces publics, la formation en ligne, lorsqu'elle est mise en œuvre dans le respect des règles d'accessibilité ${ }^{4}$, constitue parfois le seul moyen d'accéder à des compétences.

On découvre dans ce cas-là à quel point l'usage de moyens de communication visuels, qui vient se substituer à la mise en scène du professeur devant son tableau, peut se révéler excluant et contraire à l'instauration d'une relation favorable à l'apprentissage. La communication du savoir et le dialogue, transmis au moyen du texte (utilisable au travers des aides techniques spécialisées ${ }^{5}$ ) et de la voix, peuvent constituer une manière satisfaisante pour tous de développer une relation pédagogique. Privés de la vue, les acteurs du dispositif sont amenés à développer une communication basée sur le langage explicite.

La mise en scène de la situation pédagogique, considérée dans cette perspective, est interrogée quant aux véritables besoins auxquels elle est supposée répondre. S'agit-il pour le formateur de «voir faire», d'observer des comportements des personnes en formation, pour en fin de compte préserver son «contrôle» sur la relation pédagogique ? L'évaluation des résultats de l'apprentissage nécessite-t-elle de voir les apprenants et si oui, dans quels cas précis ce besoin se pose-t-il ? S'agit-il autrement dit de vérifier que chacun occupe bien sa place et assume bien son rôle : que les formés sont présents dans leur position réceptive et que le formateur assure sa représentation avec prestance ? Nous avons pu observer en effet des séances de visioconférence que les acteurs s'approprient comme un jeu, à la manière d'un talk show télévisé.

4. Le World Wide Web Consortium (W3C - <http://www.w3.org/>) diffuse les normes de présentation des contenus en ligne, destinées à rendre ceux-ci utilisables par le plus large public possible : usagers déficients sensoriels, personnes qui utilisent un ancien navigateur, qui ont des difficultés de compréhension, de mobilité, d'attention, etc.

5. Il s'agit de dispositifs à la fois matériels et logiciels qui permettent de traiter des données numériques en format texte pour les convertir en signaux tactiles Braille ou en sons restitués par une synthèse vocale. 


\section{Toucher et sentir}

Notre expérience européenne, dans le cadre de projets de développement de la FOAD, a fourni l'occasion de réfléchir de manière décentrée sur la composante culturelle de certains besoins. Lors de réunions de travail, la thématique du «toucher» s'exprime spontanément, sans la moindre arrière-pensée de la part de formateurs ou de responsables institutionnels, dès lors qu'il s'agit d'envisager les modalités de la mise à distance. Nous avons entendu à plusieurs reprises ce mot «toucher» chez des intervenants évoquant les besoins que des formateurs peuvent éprouver pour établir une «bonne" relation pédagogique. Les auteurs de ces réflexions ne semblaient pas penser que le fait de toucher réellement les apprenants pourrait constituer une transgression dans leur rôle auprès de ceux-ci ; il convient donc d'accorder à leur propos un sens figuré. L'expression métaphorique autour du toucher est en effet particulièrement fournie : on peut être « touché » par une marque de délicatesse et même "avoir quelqu'un dans la peau »! Ce choix de vocabulaire, si l'on veut bien y prêter attention, ne nous semble pas indifférent.

Il est apparu par ailleurs qu'une mise à distance intégrale de la formation (les formateurs et les participants ne se rencontrant jamais, ni «physiquement» ni par des moyens de visioconférence synchrone) ne pose pas de problème particulier dans le contexte anglo-saxon. Ici, l'éloignement géographique (à l'échelle intercontinentale) semble banalisé, si l'on en croit le succès des formations totalement en ligne (avec un tutorat asynchrone) qui y sont proposées ${ }^{6}$. En France, avec le même dispositif, nous avons pu vérifier (c'est l'objet de notre témoignage au début de cet article) que cet éloignement pouvait être assumé sans nuire au bon fonctionnement d'une formation, mais sans toutefois assouvir totalement ce besoin de « sentir » pour, comme l'exprime un des participants, « savoir à qui on a vraiment à faire ».

Dans ce contexte culturel plus latin, il semble en effet que les protagonistes de la relation pédagogique éprouvent un besoin de percevoir ce qui émane intimement de l'autre, de déchiffrer les signes non verbaux qui pourraient exprimer des aspects cachés de sa personnalité ; comme s'il fallait vérifier la cohérence des comportements ou des paroles avec les pensées secrètes, trahies par le langage du corps. Ce besoin de « sentir» semble communément valorisé comme le signe d'une intention louable d'établir une « bonne » relation pédagogique. Même si elle aboutit au constat que tel enseignant «ne peut plus sentir» tel élève, cette pulsion à vouloir percer l'intimité de l'autre est rarement questionnée.

\footnotetext{
6. Au moment où nous débutions en France notre première formation en ligne totalement à distance, nos partenaires du Royaume-Uni avaient déjà formé plus de 1000 participants selon ces modalités, en s'appuyant sur une homologation publique.
} 


\section{Ecouter et entendre}

On est particulièrement frappé de l'impérieuse nécessité de concentrer son attention lors d'une conversation avec une personne sourde. L'une d'elles nous disait à juste titre : «Mon handicap, c'est toi !», tant il est vrai qu'un manque d'attention peut induire beaucoup de frustration dans le fait d'être effectivement entendu et compris. Lorsque cette personne a appris à parler et peut lire sur les lèvres de son interlocuteur, elle ne peut assumer, en plus, l'effort de s'exprimer verbalement en étant prise dans un rythme d'interlocutions rapides, ni d'être interrompue, comme dans une discussion courante entre personnes valides. Cette nécessité d'écoute est particulièrement accentuée lorsque intervient un interprète en langue des signes. Le silence et la réceptivité doivent être totaux pendant tout le temps où la personne sourde s'exprime et durant celui de la traduction. On imagine donc sans peine le défi que constitue l'intégration de personnes sourdes dans un groupe en formation, notamment lors de phases de communication croisée entre pairs et avec le formateur. Cette difficulté de communiquer «naturellement» peut constituer une violente situation d'exclusion.

Pour les déficients auditifs qui ont eu «la chance» de pouvoir accéder à un bon niveau de maitrise du langage écrit, on peut facilement concevoir à quel point la mise à distance, avec des moyens de communication asynchrones, représente une solution adaptée. Pour les autres (valides communiquant avec des sourds), l'utilisation de moyens audiovisuels synchrones est intéressante aussi, dans la mesure où l'éloignement impose une rigueur accrue dans les processus d'écoute et de prise de parole.

Ces observations nous semblent indiquer à quel point la réalité que nous tentons d'appréhender est paradoxale et jonchée de fausses évidences. Un sourd peut écouter quelqu'un (au sens propre), de même qu'il est possible d'entendre et de comprendre un muet. De plus, cette réflexion sur l'écoute nous amène au cœur de ce que Rogers considère, avec tous ceux qui s'inspirent de son approche, comme une condition essentielle de la relation. Car il est bien question ici de relation, c'est-à-dire pas seulement de communication, de transmission de messages, mais d'interactions entre des sujets engagés affectivement. C'est pourquoi, dans le prolongement de ces témoignages, il convient de préciser de manière plus formelle et théorique ce que nous entendons par relation.

\section{« Etre en relation » : questions et définitions}

L'approche rogérienne fournit une description à la fois simple et approfondie des attitudes et comportements qui permettent d'établir un «contact réel» et de « partager des sentiments » avec l'autre, c'est-à-dire « d'être en relation » (Rogers, 1999). C'est par l'écoute et la congruence que cette relation peut s'établir, mais leur 
mise en actes, dans la situation d'éloignement physique, pose un certain nombre de problèmes que nous allons tenter d'identifier.

\section{La relation dans l'approche rogérienne}

L'écoute, dans la perspective de Carl Rogers (1999), se donne pour but de comprendre l'autre au-delà de l'expression consciente et du message explicite : « Je veux dire que j'écoute les mots, les pensées, les intonations, la signification qu'y met la personne, et même la signification qui se trouve au-delà de l'intention consciente de celui qui parle. » Elle suppose en effet de voir percer chez l'autre des signes émotionnels par lesquels il exprime en retour ce qu'il ressent dans le fait d'être entendu : « Lorsque j'entends vraiment quelqu'un et les significations qui sont importantes pour lui à ce moment, lorsque je n'entends pas seulement ses mots, mais lui-même, et que je lui fais comprendre que j'ai entendu ce que signifie pour lui son message, alors beaucoup de choses se passent. Il y a d'abord un regard plein de reconnaissance. L'autre se sent libéré. [...] Je crois qu'il devient plus disponible au processus du changement. [...] Une chose que j'ai fini par considérer presque comme universelle, c'est que lorsque quelqu'un se rend compte qu'il a été entendu en profondeur, ses yeux deviennent humides. »

Cette intensité de partage émotionnel nécessite, si l'on admet cette proposition, une proximité physique que l'on peut difficilement reconstituer au moyen d'outils de communication à distance. Le langage écrit ou parlé peut transmettre des témoignages d'écoute des mots, mais le feed-back nécessiterait une mise en scène sophistiquée (gros plans vidéo, son haute-fidélité) et un usage fortement maîtrisé des médias électroniques par tous les acteurs en communication. On peut penser que les progrès techniques rapides et l'évolution des usages culturels permettront d'atteindre cette finesse de communication dans un proche avenir.

Rogers (1999) définit ensuite la congruence: «Au lieu du mot authenticité (realness), j'ai parfois employé le mot de congruence. Par là j'entends que lorsque ce que j'expérimente objectivement se trouve au même moment présent dans ma conscience, et que tout ce qui est présent dans ma conscience l'est aussi dans ma communication, alors chacun de ces trois niveaux est en adéquation avec les autres, leur est congruent. » Il s'agit donc de mettre en adéquation ce que l'on ressent, ce que l'on pense et ce que l'on dit. Cette attitude et cette capacité consistant à se présenter tel que l'on est rencontre-t-elle des difficultés particulières dues à la mise à distance? Ces difficultés de la congruence sont essentiellement d'ordre psychologique : «Il n'y a fondamentalement aucune raison d'avoir peur lorsque je me présente tel que je suis, lorsque je m'avance sans défenses, sans armure, en étant simplement moi. [...] En outre, le fait que j'accepte de me montrer vulnérable fait apparaitre bien plus de sentiments vrais chez ceux qui sont en relation avec moi. »

On pourrait penser dans ces conditions que l'éloignement physique, loin de s'opposer à cette attitude, constituerait plutôt une aide. Etant physiquement moins 
exposé, je peux dire plus facilement ce que je ressens. Je peux aussi laisser mûrir cette expression de moi-même et prendre le temps nécessaire pour dépasser mes propres résistances. «Généralement, il y a un décalage [...] entre le moment de mon expérience et le moment de ma communication. Dans ces cas, je fais l'expérience de quelque chose, je ressens quelque chose, mais ce n'est que plus tard que j'en deviens conscient, ce n'est que plus tard que j'ose en parler, lorsque les choses sont assez refroidies pour que je puisse me risquer à en parler avec un autre. » (Rogers, 1999). On peut alors considérer que la mise à distance et la communication asynchrone permettent et facilitent même cet aspect de la relation.

La distance pose donc quelques difficultés pratiques, sans toutefois constituer un obstacle à la relation pédagogique. L'écoute et la congruence restent réalisables à distance et Rogers nous indique par quels moyens les pratiquer. Mais la question est moins de pouvoir que de vouloir engager ce type de relation. C'est sous cet angle que nous allons tenter d'approfondir notre réflexion, en proposant une définition qui, avec l'éclairage de la psychanalyse, devient plus problématique.

\section{Un processus psychologique qui assume la séparation}

Toute relation humaine est fondée et déterminée par un processus initial unionséparation - relation. Déterminée en effet, cette relation l'est par l'événement physique initial de la naissance qui va modeler les processus psychologiques d'union et de séparation intervenant dans le destin de chaque être humain (Cifali, 1994).

L'état d'union se caractérise par une totale dépendance entre des êtres non différenciés : la survie de l'un dépend de ce que chaque un ressente le même besoin. Selon l'expression de Winnicott (1975), la mère "suffisamment bonne» s'adapte presque à $100 \%$ aux besoins de son bébé, ce qui permet à ce dernier «d'avoir l'illusion que son sein à elle est une partie de lui, l'enfant ».

Toute séparation représente donc cette menace vitale de la perte : perte de ce qui apporte la sécurité et l'assurance d'une satisfaction du besoin. La séparation va pourtant amener la nécessité de relier ce qui a été séparé. La relation trouve sa place entre des Moi détachés, elle existe dans le paradoxe : ré-unir ce qui a été séparé et maintenir séparés des êtres qui vont développer leur capacité d'être seuls ${ }^{7}$. Nous parlons donc de relation en considérant ce lien paradoxal qui permet à la fois la proximité nécessaire pour vivre, agir, ressentir, penser et créer ensemble, mais aussi la distance qui admet l'autre comme non-moi et rend possible l'épanouissement de soi, différent des autres.

7. Cette expression correspond au titre d'une publication de D. W. Winnicott, «The capacity to be alone » (1958) dont les thématiques relatives au processus de maturation de l'enfant et au développement des capacités créatives sont largement développées dans l'ouvrage Jeu et réalité que nous mentionnons dans la bibliographie de cet article. 
Cette définition nous permet de mesurer l'importance des tumultes émotionnels que chaque relation met en jeu, puisqu'il s'agit à chaque fois de raviver la tension entre désir d'union et réalité (ou angoisse, ou refus) de séparation.

\section{Modèle pédagogique traditionnel et fantasme de la proximité physique}

D'un point de vue matériel et spatial, la mise en œuvre d'une formation à distance va consister à éloigner l'une de l'autre les trois instances essentielles de tout dispositif pédagogique : le formateur (ou l'enseignant), l'apprenant (on l'élève) et l'objet de l'apprentissage (pour simplifier: le savoir). Le désormais célèbre et traditionnel «triangle de Houssaye » représente d'une manière distincte et séparée ces trois instances. Pour Jean Houssaye (1988), comme pour beaucoup d'autres pédagogues après lui, il est devenu clair qu'un modèle pédagogique se constitue dans l'articulation privilégiée de deux des instances et sur l'exclusion de la troisième. Ce que nous appelons ici «modèle traditionnel » se situe donc du côté du processus « enseigner » : celui qui relie l'enseignant au savoir. Dans ce modèle, que l'on peut aussi qualifier de transmissif, l'accès au savoir par l'apprenant passe essentiellement par l'intermédiaire du formateur. Ce dernier incarne la figure sociale du dépositaire du savoir, au point d'en contrôler concrètement la diffusion, non seulement par son style pédagogique, mais aussi par sa disponibilité, ses horaires de travail, sa présence dans un lieu donné, etc. (figure 1). Dans l'hypothèse développée ici, nous proposons d'aller encore un peu plus loin dans cette idée et de considérer que, dans certaines représentations que se font des acteurs de la formation, le savoir se confond totalement avec la personne du formateur. Cette proposition peut sembler caricaturale, mais qu'en est-il dans la pratique et comment fonctionne-t-elle au plan psychologique?

Dans le modèle traditionnel en effet, le savoir est comme cette bonne nourriture que déverse le formateur, tel une mère nourricière, dans la bouche assoiffée du formé. C'est la vision que développe René Kaës (1984) pour qui, « dans la relation pédagogique traditionnelle, [...] le rapport de l'enseignant à l'objet-savoir s'établit en termes d'identification narcissique à l'objet intériorisé. L'objet devient substance, c'est l'aliment (nourriture spirituelle, substantifique moelle) ingéré et digéré devenu matière (matières d'enseignement), il est nourriture puis production même de l'enseignant. Celui-ci la possède, il en est constitué...»

C'est probablement ce registre du fantasme ${ }^{8}$, tel que le décrit Kaës, que révèle l'usage du terme de «toucher» dans la parole des professionnels de la formation. Que recherche-t-on dans la quête d'une proximité sensorielle ? Sans doute moins la chaleur physique proprement dite que l'idée réconfortante du modèle connu, éprouvé, finalement sans risque. Dans la définition du fantasme, Laplanche et

8. En psychanalyse, le fantasme est un scénario imaginaire qui figure « l'accomplissement d'un désir et, en dernier ressort, d'un désir inconscient » (Laplanche et Pontalis, 1967). 
Pontalis (1967) décrivent aussi une production du psychisme "purement illusoire qui ne résisterait pas à une appréhension correcte du réel ». C'est bien ce réel-là que nous tentons de percevoir à travers notre détour par la réflexion sur la privation sensorielle. Tel qu'il est conçu dans le modèle transmissif, le savoir s'écoule de la personne même du formateur, il émane de son propre corps ; en ce sens, il n'existe pas en tant qu'entité distincte. Ce produit se présente comme celui du formateur car il le fabrique en son sein. Le toucher s'impose donc "naturellement» puisque la transmission implique bien de prendre ou de donner le sein.

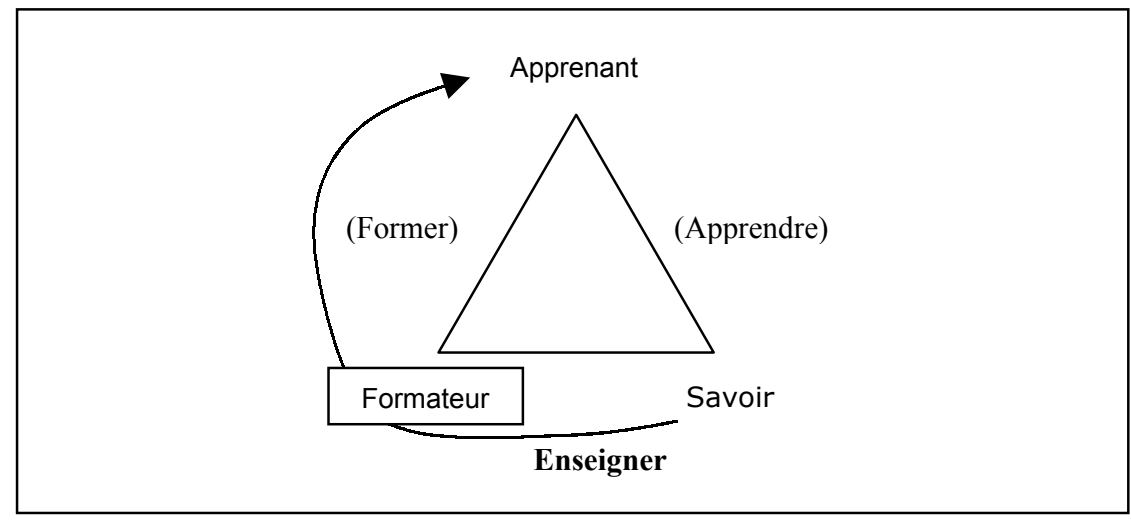

Figure 1. Modèle traditionnel dans le "triangle de Houssaye »

Dans ces conditions, en considérant ce registre fantasmatique, le triangle de Houssaye n'a plus aucune pertinence à représenter la situation pédagogique. On a à faire à des représentations englobantes : l'instance du savoir tend à se confondre avec celle du formateur et ce dernier ne peut se représenter dans une séparation avec le formé (figure 2). C'est dans la fusion, prolongeant l'enfermement dans la matrice, que se maintient le lien avec le formé. Kaës (1984) exprime encore d'une manière très imagée cette position qui «transparait dans certains fantasmes de moniteurs (hommes ou femmes) [...] Ils "portent» le groupe comme une femme enceinte, jouissant de ce plaisir d'être plein de vie [mais] inquiets aussi de les exposer à recevoir des coups, de les mettre au monde [...], de les perdre et de s'en séparer. »

Il n'est pas étonnant que la dimension «écouter» soit oubliée dans cette représentation inconsciente de la relation pédagogique. L'écoute, de la part du formateur, suppose en effet deux êtres séparés, deux Moi distincts. Seul est concevable ici le flux à sens unique qui vient alimenter le formé, en ignorant même sa demande et sa place de sujet. 


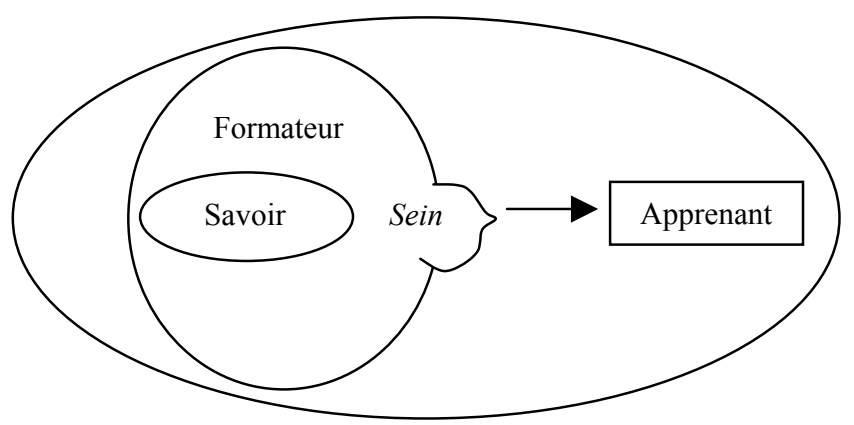

Figure 2. Représentation englobante de la relation pédagogique

\section{L'importance du « tiers » dans la mise à distance}

Dans la mise en ouvre de dispositifs de formation à distance, nous allons considérer deux types de «tiers » par rapport au couple apprenant-formateur : c'est d'une part la mise en place du savoir comme une instance séparée du formateur et, d'autre part, la possibilité de voir le rôle du formateur se transformer de celui qui transmet en celui qui accompagne.

\section{Le tiers savoir}

Avant même le développement de la formation à distance, de l'apprentissage en ligne et des systèmes de gestion électronique des contenus de formation, on a pu voir surgir les mêmes résistances face à la mise en place des centres de ressources dans le contexte de l'individualisation et de l'autoformation, dans des dispositifs tels que les « ateliers de pédagogie personnalisée » (APP).On a pu observer à quel point certaines armoires des formateurs restaient jalousement fermées et combien d'objections la perspective de mettre en place un savoir disponible pouvait soulever. Le centre de ressources trouve en effet son intérêt et son utilité dans le fait de permettre à l'apprenant un accès direct et autonome au savoir (processus «apprendre »). Ce dernier trouve là la possibilité et aussi le « risque» de chercher, d'explorer par lui-même des sources de connaissances, sans que celles-ci lui proviennent par l'intermédiaire d'un formateur. Face à cette perspective de libre accès au savoir, l'objection est double. L'apprenant est représenté dans son incapacité à trier entre les bonnes et les mauvaises ressources, à choisir celles dont il aurait vraiment besoin au bon moment. Dans cette configuration inconsciente, il est « évident» que le formateur «sait» de quel bon savoir l'apprenant a besoin et à quel moment il faut le lui apporter. La question de sa demande ne se pose même pas 
et encore moins celle de son désir. Que dire alors lorsque le centre de ressources regroupe des contenus provenant de différents auteurs ou formateurs? Et que dire surtout de la «fiabilité » de cette multitude de contenus disponibles sur internet?

Le savoir peut être pris en compte en tant que tel, comme une instance distincte, à condition de surmonter ces sentiments et ces peurs inconscients, en fonction desquels une nourriture est susceptible d'être malsaine ou empoisonnée, dès lors qu'on ne peut en contrôler l'origine, que celle-ci est autre. Alors que nous mettions en place nos premières expériences de formation ouverte et à distance, quelques années plus tôt, ce débat autour du contrôle des sources prenait une large place ; on discutait beaucoup pour déterminer si les contenus devaient être diffusés en ligne ou bien sur des cédéroms, tels de « bonnes galettes » dont l'origine serait contrôlée.

\section{Le tiers accompagnateur}

Avec la multiplication des lieux collectifs permettant l'accès à la formation à distance tels que les «points d'accès à la téléformation» $(\mathrm{P} @ \mathrm{~T})$, l'apparition d'un tiers vivant, autre que le formateur expert du contenu, vient à son tour menacer la relation quasi exclusive de ce dernier avec l'apprenant. Lorsque le formateur est éloigné du lieu où sont accessibles les ressources formatives diffusées en ligne, on trouve fréquemment des professionnels («tuteurs», «accompagnateurs») ayant pour mission d'accueillir les apprenants et de leur faciliter l'usage des techniques d'apprentissage en ligne. Ces accompagnateurs de proximité ne sont pas des spécialistes du contenu de la formation mais leur présence auprès des formés vient représenter une autre intrusion que doivent assumer les formateurs maintenant éloignés de leurs publics. Dans cette situation concrète, nous avons parfois observé une forte demande de moyens de communication synchrones, notamment la visioconférence, pour compenser cette privation de contact direct avec les formés. Ce métier d'accompagnateur de proximité est certes nouveau, mais on assiste fréquemment, lors de la mise en place de son rôle, à des négociations qui ne sont pas sans rappeler le scénario familial dans lequel, pour que tout se passe bien, Françoise Dolto (1994) dirait que « le père doit exister dans la parole de la mère ». Certaines situations de refus et de non-reconnaissance de ce tiers viennent en effet montrer toute la difficulté de dépasser cette relation duelle et exclusive de l'enseignant avec son élève. Il est possible aussi, bien sûr, que ce dispositif à trois connaisse des péripéties telles que le lien privilégié vienne s'instaurer entre l'apprenant et son accompagnateur de proximité; le formateur spécialiste, ex-maître du jeu, se retrouvant alors dans le rôle de l'intrus.

Mais cette transformation des rôles peut soulever des résistances, même si cela ne concerne qu'une seule et même personne. Le rôle de facilitateur, tel que l'envisageait Carl Rogers, constitue déjà une alternative radicale par rapport au rôle d'enseignant. Cette alternative est différente dans les postures et dans les actes, puisque l'apprentissage ne dépend plus de l'enseignement dispensé, mais de 
l'initiative dont jouit l'apprenant pour trouver des savoirs disponibles. En deçà des objections rationnelles qui s'expriment face à cette liberté il faut voir aussi, probablement, des mobiles inconscients. Ce rôle de facilitateur implique en effet une nécessaire triangulation. La relation de l'apprenant au savoir est directe et fonctionne d'une manière autonome ; le facilitateur est celui qui écoute et, de ce fait, accède à la demande de l'autre considéré comme une personne distincte.

\section{L'objet des résistances}

Comment expliquer les résistances qui se manifestent face à la mise à distance de la relation pédagogique? En nous appuyant sur la définition freudienne, nous pouvons décrire la résistance comme une force qui, chez le sujet, s'oppose à rendre conscientes certaines représentations inconscientes (Freud, 1981). Sortir d'une certaine confusion entretenue dans le modèle transmissif va nécessiter une prise de recul, un mouvement visant à prendre conscience du fonctionnement de cette relation pédagogique. Ainsi des questions simples comme: «qu'en est-il de ma position par rapport au savoir?», "quelle est ma position vis-à-vis des apprenants?», peuvent provoquer certaines gênes ou certains malaises. De telles questions sont certes difficiles sur un plan théorique, mais le savoir élaboré par les sciences humaines ne permet-il pas d'y répondre ? Or ce savoir lui-même suscite autant d'espérance que de méfiance (Cifali, 1994); il est celui des experts et les praticiens ne s'y reconnaissent pas toujours, loin s'en faut. Le triangle pédagogique constitue, semble-t-il, une représentation suffisamment simple et symbolique pour qu'à la fois les uns et les autres puissent y projeter leurs représentations.

Dans la perspective que nous avons ébauchée, chaque sommet du triangle intervient comme un tiers pour les deux autres. Ce tiers, être vivant ou simple miroir, agit comme un témoin ou un révélateur du lien qu'entretiennent les deux autres (s'ils veulent bien l'entendre). Ainsi par exemple, dans un modèle fortement dominé par le processus «apprendre», le formateur dit à l'autodidacte : «voilà comment tu réussis ou tu échoues à t'approprier ce savoir. " Dans le modèle où prédomine le processus « enseigner », l'apprenant pourrait dire : « que fais-tu de ma personnalité, de ma demande et de ma façon d'apprendre? » Cette troisième instance apparaît alors comme menaçante, dans la mesure où elle vient percuter une structure inconsciente fondée sur la dualité, la fusion et l'exclusion. En tant que facteur de prise de recul, de distanciation (Bougès, 2002), elle est propice à la construction du savoir. Mais la thématique de la distance en tant qu'éloignement physique semble plutôt cristalliser des représentations inconscientes qui évoquent les douleurs de la séparation et la terreur de l'abandon. Cette idée d'abandon ne vient-elle d'ailleurs pas servir de repoussoir vis-à-vis de la formation à distance ? «Combien d'apprenants on-t-ils abandonné leur formation?» «Que faut-il faire pour qu'ils ne se sentent pas abandonnés? " Ce sont des questions qui se posent souvent et avec gravité. Il semble bien que l'on trouve là, pour beaucoup de projets, un mur psychologique infranchissable. 


\section{Conclusion : la mise à distance facilite-t-elle la relation ?}

En introduction, il a été question du défi que représente la confrontation aux résistances, notamment celles que suscite la mise à distance de la relation pédagogique. Défi pour la pensée, selon Mireille Cifali (1994), mais pour les acteurs du terrain de la formation, ce défi peut être celui de toute une vie professionnelle. Les progrès techniques réalisent parfois des bonds qualitatifs très importants, comme c'est le cas aujourd'hui avec le numérique. Alors la réponse est toute en nuances : oui, la distance physique peut parfois aider à trouver une juste distance psychologique. Celle-ci dépend avant tout de ce que veulent et ce que peuvent les acteurs, psychologiquement et culturellement. Les progrès nourrissent les forces en présence, autant les résistances que les espérances. Dans notre champ d'action, il nous a semblé que les composantes psychologiques de ces différentes forces méritaient d'être mieux explorées. Nous mesurons cependant toute l'ambition de cette entreprise. Nous avons employé notre conviction et notre réflexion à tenter des explications qui restent au stade d'hypothèses et mériteraient des traitements plus systématiques, dans le cadre de travaux de recherche. Les témoignages et observations présentés demanderaient à être confrontés et comparés. De plus, l'angle de vue psychologique que nous avons privilégié est loin de tout pouvoir expliquer, tant il est vrai que les enjeux d'argent et de pouvoir, les logiques institutionnelles, ont bien évidemment un poids déterminant dans l'univers de la formation.

\section{Bibliographie}

Bernard M., Penser la mise à distance en formation, Paris, L'Harmattan, 1999.

Bougès L.-M., Accompagnement et autoformation (Proximité - distance du formateur dans une Formation Ouverte en Réseau), Mémoire de DESS de Formateur consultant en Sciences et Techniques, Université de Pau, 2002.

Cifali M., Le lien éducatif : contre-jour psychanalytique, Paris, PUF, 1994.

Collectif de Chasseneuil, Accompagner des formations ouvertes, Paris, L'Harmattan, 2001.

Dolto F., Tout est langage, Paris, Gallimard, 1994.

Freud S., Essais de psychanalyse, Paris, Payot, 1981.

Houssaye J., Le triangle pédagogique, Bern, Peter Lang, 1988.

Kaës R., Fantasme et formation, Paris, Bordas, 1984.

Laplanche J., Pontalis J.-B., Vocabulaire de la psychanalyse, Paris, PUF, 1967.

Marc E., Picard D., Relations et communications interpersonnelles, Paris, Dunod, 2000.

Pagès M., La vie affective des groupes. Paris, Dunod, 2002.

Rogers C., Liberté pour apprendre, Paris, Dunod, 1999.

Winnicott D. W., Jeu et réalité, Paris, Gallimard, 1975. 